Hydrology and Earth System Sciences, 8(5), 1001-1007 (2004) C EGU

\title{
Technical Note: \\ The representation of rainfall drop-size distribution and kinetic energy
}

\author{
Neil I. Fox \\ Department of Soil, Environmental and Atmospheric Sciences373 McReynolds Hall, University of Missouri - Columbia, Columbia, MO 65203, USA
}

Email: foxn@missouri.edu

\begin{abstract}
To relate observed rainfall rates $(R)$ to the kinetic energy flux (E) that affects soil erosion it is necessary to develop relationships between the two. This paper explores theoretical E-R relationships based on gamma distributions of drop size. The relationship is poorly defined unless assumptions are made about changes in the shape of the drop-size distribution (DSD) with rainfall rate. The study suggests that the assumption of an exponential DSD leads to overestimation of kinetic energy flux. Further, incorporation of a horizontal component of kinetic energy allows for a clearer relationship between kinetic energy and rainfall intensity to be defined, but a question remains regarding the most appropriate definition of the horizontal component of drop velocity.
\end{abstract}

Keywords: drop-size distribution, drop kinetic energy, soil erosion

\section{Introduction}

The quantity of soil that is lost during a rainfall event is a function of the kinetic energy of the rain that impacts the soil. As rain consists of a spectrum of drop sizes, the kinetic energy is dependent upon the nature of the distribution of those sizes. In particular, larger drops have both greater mass and vertical terminal velocity such that a disproportionate amount of erosion results from the action of a small number of large drops. Soil erosion calculations such as those used in the WEPP model (Flanagan et al., 2001) and in RUSLE (Reynard et al., 1997) are based upon models of the distribution of raindrop sizes that date back to the 1940s (Laws and Parsons, 1943; Marshall and Palmer, 1948). These, in turn, are based on small samples collected using flour pellet or blotting paper methods. Video disdrometers, able to increase vastly the sample sizes of drop distributions, have revealed that the shape of those distributions follows forms other than those proposed by Marshall and Palmer (1948). Based on disdrometer observations, Steiner and Smith (2000) conducted an extensive survey of the relationships between the three variables, measured radar reflectivity, rainfall rate and kinetic energy (KE). They found that, while the relationship between $\mathrm{E}$ and $\mathrm{R}$ was well characterised by a linear equation, there was a great deal of scatter and, therefore, uncertainty in the form of the relationship. A similar conclusion was reached by Parsons and Gadian (2000) in a theoretical study of DSD variation and soil detachment.

Studies of rainfall using dual-polarisation weather radars and disdrometers have also revealed that the typical parameterisation of the drop-size distribution (DSD) is inaccurate and that natural rain DSDs can take different forms (Ulbrich and Atlas, 1998; Illingworth and Blackman, 2002). Generally the DSD takes the form of a gamma distribution of which the Marshall-Palmer is a special case. As shown below, the gamma distribution is defined by three variables which are not independent, but it is difficult to define the dependency (Testud et al., 2001; Uijlenhoet, 2001).

As rainfall rate is the regular observation used to assess the potential soil loss, this paper explores the theoretical relationship between measured rainfall rate and the rain kinetic energy flux (the factor that controls soil erosion). A potential exploitation of remote sensing technology and of 
weather radar in particular, could be in the areal assessment of soil erosion due to heavy rain. To achieve this requires an accurate representation of the DSD and the relationship between R and E. Typically, laboratory experiments using rainfall simulators are performed in such a way that the simulator produces a DSD that replicates what is assumed to exist in natural rain (Regmi and Thompson, 2002). However, most of these assumptions also use the MarshallPalmer DSD rather than a gamma distribution. Therefore, there is a need to investigate the difference between simulated and natural rain, in terms of the energy flux, to extend, accurately, the results of simulator studies to the natural environment.

Further to this, all previous studies of rainfall kinetic energy and its application to soil erosion problems implicitly assume that the rain falls vertically. Certainly this is the case in simulator studies where the generation of non-vertical fall trajectories is not practical. However, natural rain almost always has some component of horizontal velocity and, in some instances, this is likely to be greater than the terminal velocity of the drops (Gunn and Marshall, 1955; Lack and Fox, 2003).

The exponential form of the DSD described by Marshall and Palmer (1948) is

$$
N(D)=N_{0} \exp (-\Lambda D)
$$

in which $\mathrm{N}(\mathrm{D})$ is the number concentration $\left(\mathrm{m}^{-3}\right)$ of drops of diameter $\mathrm{D}$ (in $\mathrm{mm}$ ), $\mathrm{N}_{0}$ is the total drop concentration and $\Lambda$ (in $\left.\mathrm{mm}^{-1}\right)$ is a parameter related to the median drop diameter of the size spectrum:

$$
\Lambda=3.67 / D_{0}
$$

where $\mathrm{D}_{0}$ is the median drop size diameter denoting the drop size for which half the liquid water volume of the drops is contained in drops smaller, and half contained in drops larger than this diameter.

However, it is now recognised that the DSD of natural rain is better represented by the gamma distribution (Testud et al., 2001):

$$
N(D)=N_{T} D^{\mu} \exp (-\Lambda D)
$$

The value of $\mu$, the order of the gamma distribution, can take values between 0 (the exponential Marshall-Palmer distribution) and 15 but, generally, is found toward the bottom of this range (Illingworth and Blackman, 2002). In this case $\Lambda$ takes a value given by

$$
\Lambda=(3.67+\mu) / D_{0}
$$

To make calculations consistent, (3) must be normalised such that the total number of drops found by its integration is independent of the value of $\mu$. In the revised version of (3),

$$
N_{T}=N_{0} \frac{\Gamma(\mu+1)}{\Lambda^{\mu+1}}
$$

If DSDs for different values of $\mu$ are plotted, one obtains the curves shown in Fig. 1. These curves are for $\mathrm{D}_{0}=1.5 \mathrm{~mm}$ and $N_{T}=8000 \mathrm{~m}^{-3}$, which are typical values that are in agreement with the formulation of Marshall and Palmer (1948).

These curves show the effect of different formulations on the DSD. As the value of $\mu$ is increased, the concentration of large droplets is reduced, while the modal drop size increases. Overall, this leads to a narrower DSD which one would expect to reduce the total kinetic energy flux of the falling population of drops, as this is most sensitive to the number of large drops. In this way $\mu$ can be considered a 'shape factor' for the DSD. The major contribution to the total kinetic energy of the rain is provided by a relatively small number of larger drops. However, the increased and improved observations of natural rain have led to the conclusion that there are fewer of these drops present than previously thought. The gamma distribution used to represent the DSD is narrower than the exponential and contains the assumption that there are fewer large drops in the rain. This implies that calculations of KE based on exponential DSDs will overestimate the KE imparted by falling rain. Furthermore, laboratory based studies of soil erosion using equipment designed to replicate an exponential DSD will produce greater soil losses than would be expected in natural rain. In particular, it is believed that the Marshall-

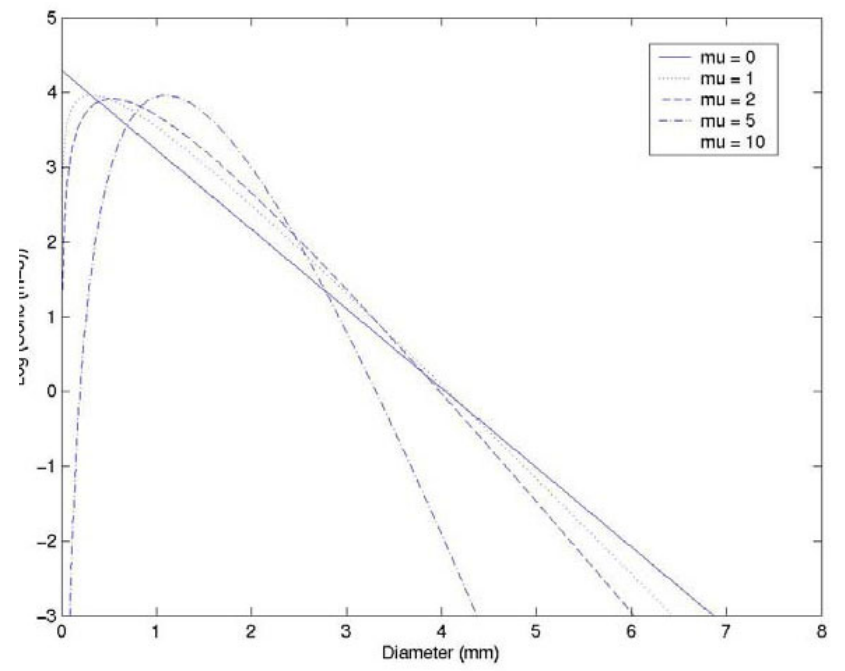

Fig. 1. Drop-size distributions for different values of $\mu$. 
Palmer DSD is appropriate for low $\mathrm{R}$, but $\mu$ increases with $\mathrm{R}$.

The objective of this theoretical study is to examine the difference between the kinetic energies produced by the different assumptions regarding the DSD. The results could be used to estimate corrections to laboratory studies when required.

This note demonstrates the impact that this change in the formulation of the DSD can make to estimates of kinetic energy and hence of soil erosion. Also, it examines the impact of including the horizontal component of momentum of falling drops, due to wind, on the overall KE of the DSD, under the differing assumptions regarding the DSD.

\section{Kinetic energy of a distribution of raindrops falling vertically}

The kinetic energy of a falling drop is given by

$$
E=\frac{1}{2} m v^{2} .
$$

where $m$ is the mass of the drop:

$$
m=\frac{\pi}{6} \rho D^{3}
$$

and $\rho$ is the density of water. The rainfall rate due to the falling population of drops at terminal velocity $v(D)$ in $\mathrm{m} \mathrm{s}^{-1}$ is then given by

$$
R=\frac{\pi}{6} \int N(D) D^{3} v(D) d D
$$

and the instantaneous kinetic energy of the rainfall is

$$
E=\frac{\pi \rho}{6} \int N(D) D^{3}[v(D)]^{2} d D
$$

The drop energy flux (rate of kinetic energy transfer) is the variable of greatest interest in erosion studies and this is given by

$$
E=\frac{\pi \rho}{6} \int N(D) D^{3}[v(D)]^{3} d D
$$

In each case, having specified $N(D)$, one can complete the integral and find the rainfall rate and kinetic energy, having assumed a formulation for the terminal drop velocity. For this work, the formulation from Ulbrich (1983) is used:

$$
v(D)=3.78 D^{0.67}
$$

This single equation, in contrast to others that use different formulae for different ranges of $D$ (Beard, 1976; Rogers and Yau, 1992), has been shown to be reasonably accurate across the range of $D$ and makes the computation straightforward. Of course, $v(D)$ may be affected by many factors, such as air density, turbulence and vertical air motions. These are ignored in this study.

Substituting (11) and the normalised version of (3) into (10) and solving the integral produces

$$
E=3.78^{3} \frac{\pi \rho}{6} N_{0} \frac{\Gamma(6+\mu)}{\Lambda^{(\mu+6)}}
$$

Using the same procedure with (8) gives

$$
R=3.78 \frac{\pi}{6} N_{0} \frac{\Gamma(4.67+\mu)}{\Lambda^{(\mu+4.67)}}
$$

To assess the relationship of the kinetic energy to the rainfall rate, the drop-size distribution has to be specified through the three parameters $N_{T}, D_{0}$, and $\mu$.

Figure 2 shows an example of the different values of $\mu$ on the relationship between rainfall rate and droplet energy flux for a fixed value of $D_{0}$. In the case of each line, the total drop concentration is varied from 1000 to $10000 \mathrm{~m}^{-3}$. Here it can be seen that, for the same rainfall rate, the lower values of $\mu$ produce higher droplet energy fluxes as there are more large drops with high kinetic energy.

Figure 3 presents the results of varying both $D_{0}$ and $N_{T}$ for the different values of $\mu$. Sample results are shown for rainfall rates up to $150 \mathrm{~mm} \mathrm{hr}^{-1}$, which can be considered as the extreme rainfall rate sustainable for very short periods of time. In this case, the relationships between the kinetic energy and rainfall rate are not simple and the same relationship may be produced by different DSD shapes. The general trend remains the same; higher values of $\mu$ produce lower kinetic energy fluxes for the same rainfall rate, as the population of drops covers a narrow spectrum of sizes and

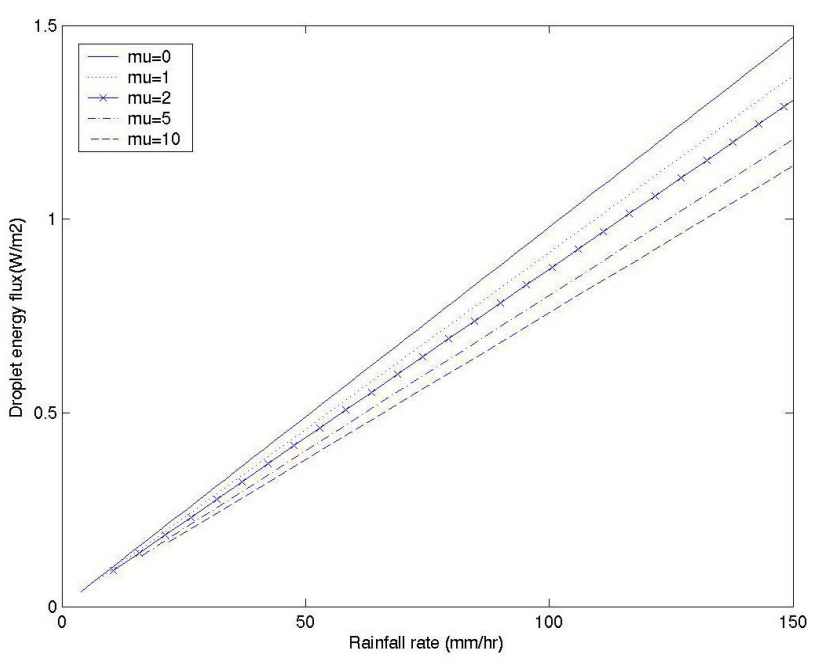

Fig. 2. Examples of different relationships between rainfall rate and droplet energy flux for $D_{0}=1.5 \mathrm{~mm}$. 


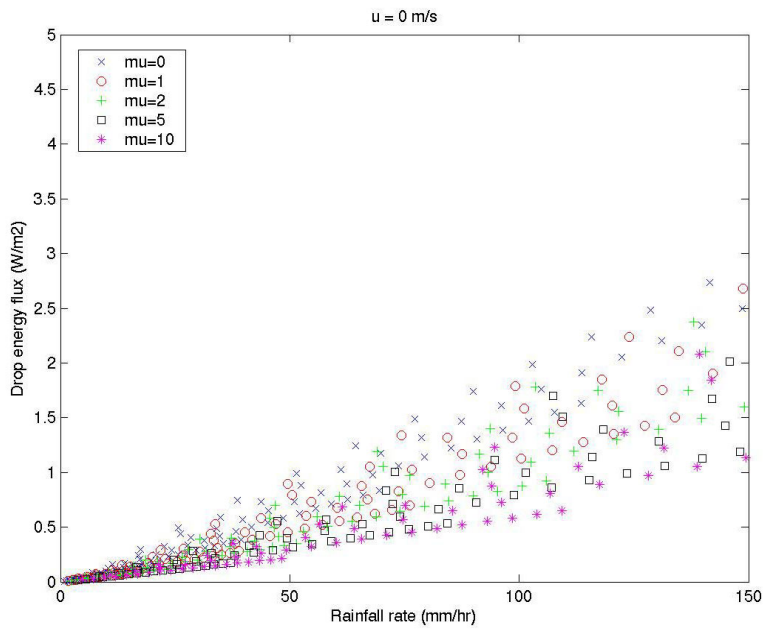

Fig. 3. Comparison of kinetic energy and rainfall rates for different values of $\mu$. [Crosses are results for $\mu=0$; circles are for $\mu=1$; squares are for $\mu=2$; pluses are for $\mu=5$; asterisks are for $\mu=$ 10.]

contains fewer large drops.

The results show good agreement with the experimental observations of Steiner and Smith (2000), including a similar linear relationship between $\mathrm{E}$ and $\mathrm{R}$. The theoretical scatter shown here appears greater than that of the observed data and this may be a result of natural rain requiring particular DSD shapes for high intensities to be achieved. That is, in natural rain, high intensities are found only with certain DSDs (values of $\mu$ ) while, in lighter rain, a wide variety of values of DSDs is possible.

Cerro et al. (1998) found that assuming a lognormal DSD produces the best fit for their observed $E-R$ relationship. However, it could also be argued that using gamma distributions that increase in order (including $\mu=0$ for low

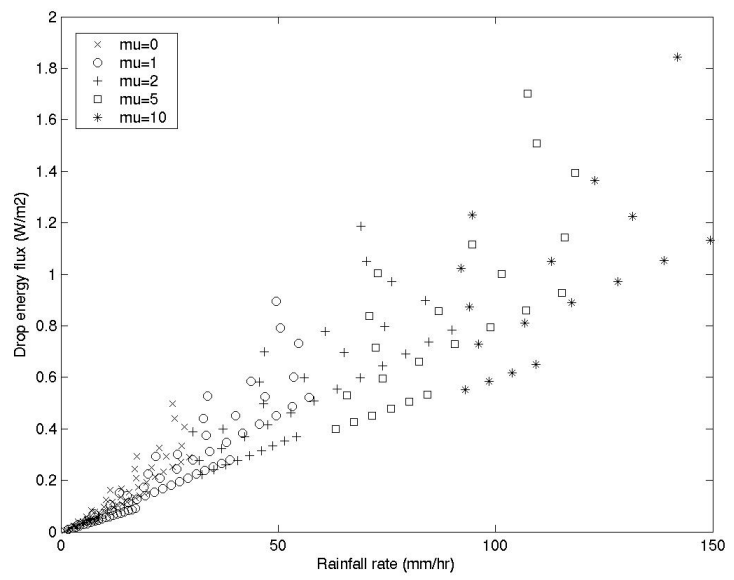

Fig. 4. As Fig..2, but with values of $\mu$ constrained to rainfall rate bands. In this case $\mu=0$ is used for $R<30 \mathrm{~mm} \mathrm{~h}^{-1}, \mu=1$ is used for $R<60 \mathrm{~mm} \mathrm{~h}^{-1}, \mu=2$ is used for $30<R<90 \mathrm{~mm} \mathrm{~h}^{-1}, \mu=5$ is used for $60<R<120 \mathrm{~mm} \mathrm{~h}^{-1}$, and $\mu=10$ is used for $R>90 \mathrm{~mm} \mathrm{~h}^{-1}$. These bounds were selected arbitrarily.
R) would yield similar results. As an example, Fig. 4 is a reconstruction of Fig. 2 with each sample of $E-R$ points restricted to specific (arbitrary) rainfall intensity bands. In this case, the E-R relationships appear closer to those presented by Steiner and Smith (2000) and Cerro et al. (1998). It is also possible that higher rates constrain the DSD further, in terms of $D_{0}$ for example.

\section{Kinetic energy of a distribution of raindrops with horizontal component of velocity}

Under normal conditions, rain does not fall vertically but has a component of horizontal velocity imparted by the wind which is generally of the same order as the vertical velocity of the drops. The total kinetic energy flux $\left(E_{T}\right)$ of a falling population of raindrops under the influence of a horizontal wind of velocity $u$ is given by

$$
E_{T}=\frac{\pi \rho}{6} \int N(D) D^{3}[v(D)]^{3} d D+\frac{\pi \rho}{6} \int N(D) D^{3} u^{2} v(D) d D
$$

Here, it is assumed that all drops of all diameters have the same horizontal component of velocity determined by the wind at some level above the ground. In reality, the wind near the surface will be lower than that at elevation and the drops will suffer differential horizontal deceleration as the smaller drops experience a greater drag than the larger drops. This differential deceleration is difficult to quantify and is dependent upon complex winds near the surface, so this simplistic assumption is necessary. Completion of the integral gives

$$
E_{T}=3.78^{3} \frac{\pi \rho}{6} N_{0} \frac{\Gamma(6+\mu)}{\Lambda^{(\mu+6)}}+3.78 \frac{\pi \rho}{6} N_{0} u^{2} \frac{\Gamma(4.67+\mu)}{\Lambda^{(\mu+4.67)}}
$$

or

$$
E_{T}=E+u^{2} \rho R
$$

Following the same procedure as described previously, $\mu$ was fixed and values of $D_{0}$ and $N_{T}$ were varied to produce samples of rainfall rate and kinetic energy for a variety of wind velocities. Figure 1 can be considered the case for $\mathrm{u}=0 \mathrm{~m} \mathrm{~s}^{-1}$. Figures 5, 6, 7 and 8 show the results for wind velocities of 5, 8, 10 and $12 \mathrm{~m} \mathrm{~s}^{-1}$ respectively.

Naturally, the droplet energy flux increases with increasing wind speed and, as that speed increases, the component of kinetic energy due to horizontal motion begins to dominate. However, coupled with this is the effect that the relationship 


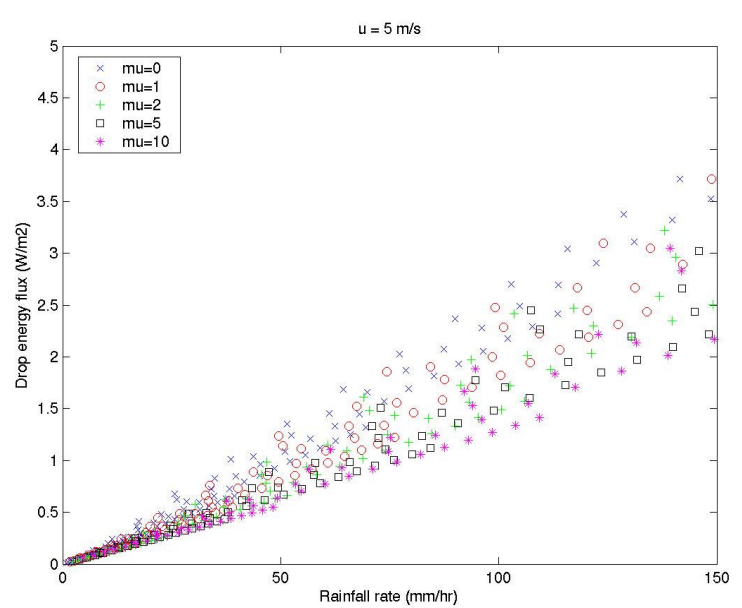

Fig. 5. Relationship between $R$ and $E$ for $u=5 \mathrm{~m} \mathrm{~s}^{-1}$.

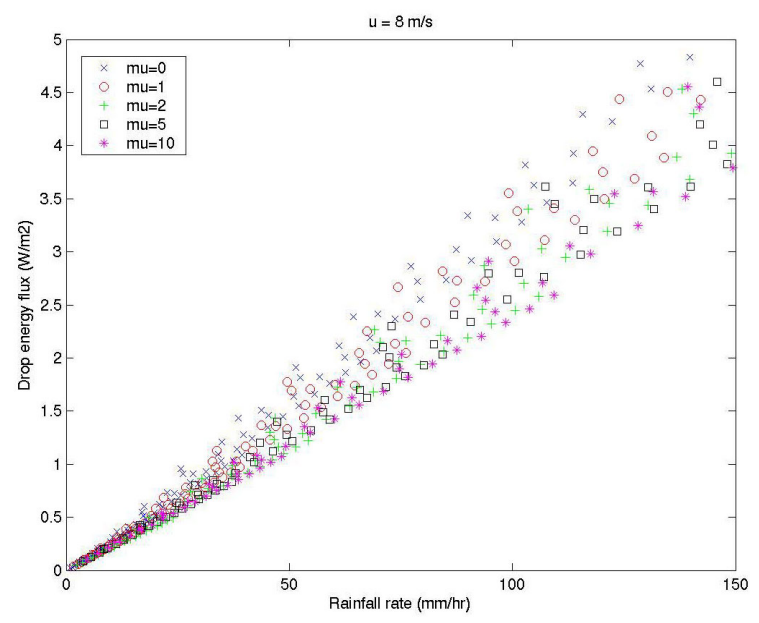

Fig. 6. Relationship between $R$ and $E$ for $u=8 \mathrm{~m} \mathrm{~s}^{-1}$.

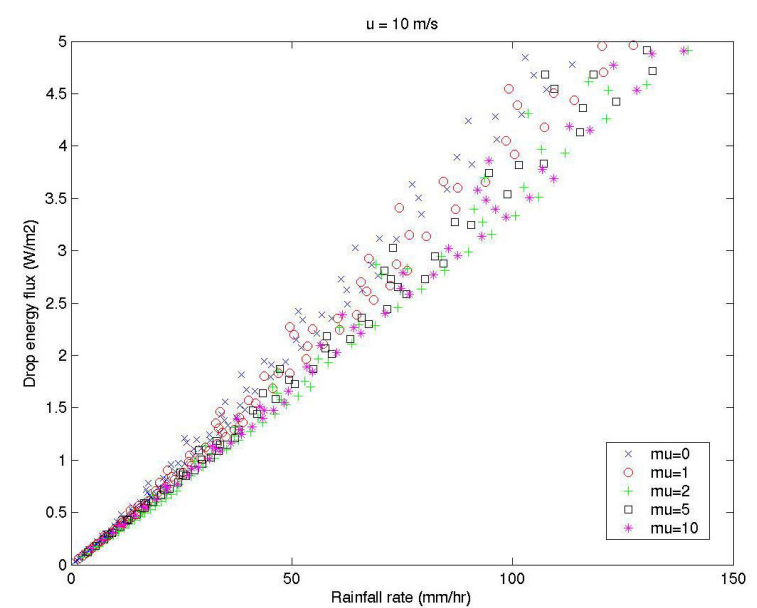

Fig. 7. Relationship between $R$ and $E$ for $u=10 \mathrm{~m} \mathrm{~s}^{-1}$.

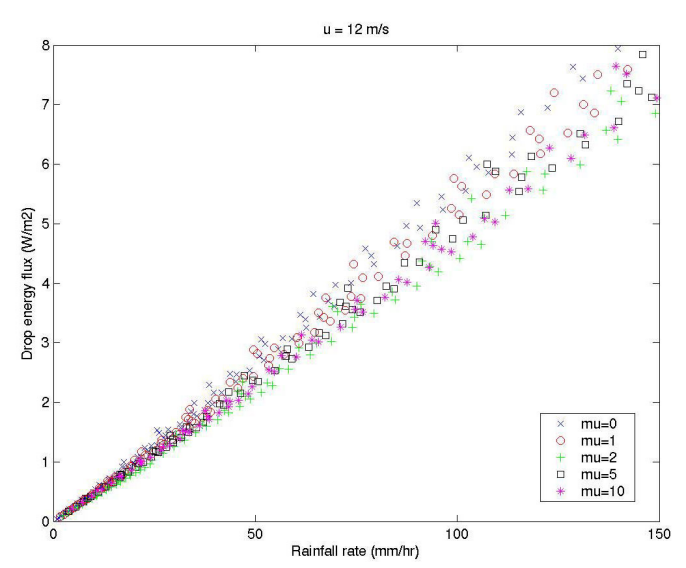

Fig. 8. Relationship between $R$ and $E$ for $u=12 \mathrm{~m} \mathrm{~s}^{-1}$.

between $E$ and $R$ becomes more defined and less dependent upon $\mu$, especially at low rainfall rates. For the highest values of u used in this study, the relationship between $E$ and $R$ appears to be linear and is defined clearly up to rainfall rates of $100 \mathrm{~mm} \mathrm{hr}^{-1}$.

From these plots, the uncertainty in a value of $E_{T}$ derived from an observed rainfall appears to increase with increasing $R$, in contrast with the findings of Steiner and Smith (2000). This may well reflect the fact that lower intensity rates of rainfall can result from a wide range of combinations of $D_{0}$, $N_{T}$ and $\mu$, whereas really heavy rainfalls can be generated only by a smaller range of possible values. The figures generated herein assume that all rainfall rates can be generated from any suitable DSD but the evidence is that this is not the case. If one assumes that higher rainfall rates cannot result from DSDs with $\mu<5$, then a theoretical figure

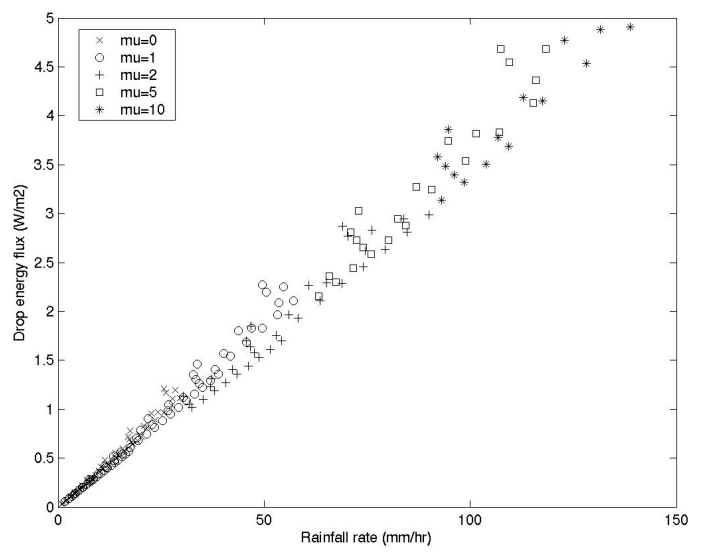

Fig. 9. As Fig. 7 for $u=10 \mathrm{~m} \mathrm{~s}^{-1}$, but with values of $\mu$ constrained to rainfall rate bands. In this case $\mu=0$ is used for $R<30 \mathrm{~mm} \mathrm{~h}^{-1}, \mu=$ 1 is used for $R<60 \mathrm{~mm} \mathrm{~h}^{-1}, \mu=2$ is used for $30<R<90 \mathrm{~mm} \mathrm{~h}^{-1}, \mu$ $=5$ is used for $60<R<120 \mathrm{~mm} \mathrm{~h}^{-1}$, and $\mu=10$ is used for $R>90$ $\mathrm{mm} \mathrm{h} h^{-1}$. These bounds were selected arbitrarily. 
such as Fig. 9 can be produced. Here, with $u=10 \mathrm{~m} \mathrm{~s}^{-1}$, there is much less scatter, particularly for higher values of $R$.

Using Eqn. (3), and the assumption that drops retain the horizontal component of motion due to some representative wind speed down to the surface, it is notable that a $2 \mathrm{~mm}$ diameter drop will have a fall angle of $50^{\circ}$ when the wind is $5 \mathrm{~m} \mathrm{~s}^{-1}$, and a fall angle of $3^{\circ}$ when the wind is $10 \mathrm{~m} \mathrm{~s}^{-1}$. Similarly a $3 \mathrm{~mm}$ drop would have fall angles of $58^{\circ}$ and $38^{\circ}$ with wind speeds of 5 and $10 \mathrm{~m} \mathrm{~s}^{-1}$ respectively, while a $5 \mathrm{~mm}$ drop would have fall angles of $66^{\circ}$ and $48^{\circ}$. A $10 \mathrm{~m} \mathrm{~s}^{-1}$ wind would be considered a high wind at the surface. In natural meteorological conditions, it is not uncommon for high winds to accompany heavy rains, and much soil is lost in consequence of the extreme rainfall conditions produced by tropical storms. In such cases, the wind velocities used in this study would be considered low.

\section{Discussion}

The study presented here demonstrates no clear relationship between observed rainfall rate and droplet kinetic energy flux. Furthermore, the assumption of a particular shape to the DSD can bias estimates of soil loss. In particular, the use of the exponential DSD proposed by Marshall and Palmer (1948), although generally accepted as appropriate as a representation for reasonably low intensity mid-latitude stratiform rains, overestimates the number of large drops present in heavier rains and, hence, the kinetic energy of that rain. However, if the correct form of the DSD for the rain is specified, then the bias can be corrected.

As the horizontal wind velocity increases, the kinetic energy of the rain due to horizontal motion increases and dominates in comparison with the vertical component of the kinetic energy. However, the impact of the rain on the soil surface is complex and is not a simple function of the kinetic energy, especially as the rain impacts the surface at an angle. In these circumstances, the quantity of soil dislodged depends also on the impact angle (which includes the slope of the surface and the slope relative to the fall angle of the drops), the soil type, structure, moisture content and many other factors. Also, drops of different sizes will impact the surface at different angles; the largest drops with the highest terminal velocity, will strike the surface with a higher perpendicular velocity than smaller drops.

Many simplifications have been made in this work. These results ignore the effect of changes in vertical velocity caused by the vertical motion of the air (updrafts and downdrafts). Other formulations of the terminal velocity have been suggested and no dependence on air density is provided. However, errors in the assumptions of drop velocity are unavoidable in natural rain, unless those velocities are observed directly, which is impractical in any areal survey. Doppler radar also measures the horizontal velocity of the falling drops, albeit at elevation, and this could be used to find the total kinetic energy of the falling drops. However, the determination of the appropriate horizontal component of motion is difficult and also dependent on the DSD.

The results show the importance of portraying, accurately, the shape of the DSD in both estimates of natural soil erosion and simulator experiments. As the simulators tend to produce either exponential DSDs or DSDs that only approximate the desired shape, then the findings presented here could be used to adjust the estimates of soil loss from simulators. The soil detachment process is so complex that it must be studied under controlled conditions in simulators; yet, to extend these results to realistic estimates of areal soil erosion (as can be made using radar observations of rainfall), laboratory results must be compared with observations of natural rain and with these theoretical relationships to reconcile the differences between laboratory studies and field observations. This is particularly important in respect of the horizontal component of velocity and work is needed to quantify the true horizontal velocity at the point of impact on the surface.

\section{Acknowledgements}

The author would like to thank Dr. Allen Thompson and Dr. Clark Gantzer for their patient and informative discussions that contributed to this work.

\section{References}

Beard, K.V., 1976. Terminal velocity and shape of cloud and precipitation drops aloft. J. Atmos. Sci., 33, 851-864.

Cerro, C., Bech, J., Codina, B. and Lorente, J., 1998. Modeling rain erosivity using disdrometer techniques. Soil Sci. Soc. Amer. J., 62, 731-735.

Flanagan, D.C., Ascough II, J.C., Nearing, M.A. and Laflen, J.M., 2001. The Water Erosion Prediction Project (WEPP) Model. In: Landscape Erosion and Evolution Modeling, R.S. Harmon and W.W. Doe III (Eds.), Kluwer, Norwell, MA, USA. 51pp.

Gunn R.E.S. and Marshall, J.S., 1955. The effect of wind drift on falling precipitation. J. Atmos. Sci., 12, 339-349.

Illingworth, A.J. and Blackman, T.M., 2002. The need to represent raindrop size spectra as normalized gamma distributions for the interpretation of polarization radar observations. J. Appl. Meteorol., 41, 286-297.

Lack, S.A. and Fox, N.I., 2003. Correcting radar derived rainfall rates at the surface using Doppler velocities. Preprints $31^{\text {st }}$ Conf. on Radar Meteorology. Amer. Met. Soc., Boston, MA, USA. 229-232.

Laws, J.O. and Parsons, D.A., 1943. The relation of raindrop size to intensity. Trans. Amer. Geophys. Un.., 24, 452-460. 
Marshall, J.S. and Palmer, W.McK., 1948. The distribution of raindrops with size. J. Meteorol., 5, 165-166.

Parsons, A.J. and Gadian, A.M., 2000. Uncertainty in modeling the detachment of soil by rainfall. Earth Surf. Process. Landf., 25, 723-728.

Regmi, T.P. and Thompson, A.L., 2000. Rainfall simulator design for laboratory studies. Appl. Eng. Agr. J.- ASAE, 16, 641-647.

Renard, K.G., Foster, G.R., Weesies, G.A., McCool, D.K. and Yoder, D.C., 1997. Predicting soil erosion by water: A guide to conservation planning with the Revised Universal Soil Loss Equation (RUSLE). U.S. Department of Agriculture, Agricultural Handbook No. 703.

Rogers, R.R. and Yau, M.K., 1992. A short course in cloud physics. Pergamon Press, Oxford, UK. 290pp.

Steiner, M. and Smith, J.A., 2000. Reflectivity, rain rate and kinetic energy flux relationships based on raindrop spectra. J. Appl. Meteorol., 39, 1923-1840.
Testud, J., Oury, S., Black, R.A., Amayenc, P. and Xiankang, D., 2001. The concept of "normalized"distribution to describe raindrop spectra: a tool for cloud physics and cloud remote sensing. J. Appl. Meteorol., 40, 1118-1140.

Uijlenhoet, R., 2001. Raindrop size distributions and radar reflectivity-rain rate relationships for radar hydrology. Hydrol. Earth Syst. Sci., 5, 615-627.

Ulbrich, C.W., 1983. Natural variations in the analytical form of the raindrop size distribution. J. Clim. Appl. Meteorol., 22, 17641775.

Ulbrich, C.W. and Atlas, D., 1998. Rainfall microphysics and radar properties: analysis methods for drop size spectra. J. Appl. Meteorol., 37, 912-923. 